\title{
ALS Superbend Magnet System
}

\author{
J. Zbasnik ${ }^{\dagger}$, S. T. Wang ${ }^{\dagger \dagger}$, J. Y. Chen ${ }^{\dagger \dagger}$, G. J. DeVries ${ }^{\dagger}$, R. DeMarco ${ }^{\dagger}$, M. Fahmie ${ }^{\dagger}$, A. Geyer ${ }^{\dagger}$, M. A. Green ${ }^{\dagger}$, J. \\ Harkins $^{\dagger}$, T. Henderson ${ }^{\dagger}$, J. Hinkson ${ }^{\dagger}$, E. H. Hoyer ${ }^{\dagger}$, J. Krupnick $^{\dagger}$, S. Marks ${ }^{\dagger}$, F. Ottens ${ }^{\dagger}$, J. A. Paterson ${ }^{\dagger}$, P. \\ Pipersky ${ }^{\dagger}$, G. Portmann ${ }^{\dagger}$, D. A. Robin ${ }^{\dagger}$, R. D. Schlueter ${ }^{\dagger}$, C. Steier ${ }^{\dagger}$, C. E. Taylor ${ }^{\dagger}$, R. Wahrer ${ }^{\dagger \dagger}$
}

\section{SYSTEM REQUIREMENTS AND DESIGN}

\begin{abstract}
The Lawrence Berkeley National Laboratory is preparing to upgrade the Advanced Light Source (ALS) with three superconducting dipoles (Superbends). In this paper we present the final magnet system design which incorporates $R \& D$ test results and addresses the ALS operational concerns of alignment, availability, and economy. The design incorporates conduction-cooled Nb-Ti windings and HTS current leads, epoxy-glass suspension straps, and a Gifford-McMahon cryocooler to supply steady state refrigeration. We also present the current status of fabrication and testing.
\end{abstract}

Index Terms-Superconducting magnet, conduction cooled, cryocooler

\section{INTRODUCTION}

The Advanced Light Source (ALS) at the Lawrence Berkeley National Laboratory (LBNL) is a national user facility that produces high brightness synchrotron radiation in the vacuum ultraviolet $(6 \mathrm{eV}$ to $6 \mathrm{keV})$ and soft $\mathrm{x}$-ray $(>6 \mathrm{keV})$ regimes. The approximately 200 meter circumference, $1.9 \mathrm{GeV}$, ALS electron storage ring consists of twelve cells; each cell has three 1-m long combinedfunction gradient bending magnets. Each bending magnet generates photons with a critical energy of $3.1 \mathrm{keV}$, which are delivered to users via 48 ports.

A study [1] commissioned to consider ways of increasing the photon energy concluded that an attractive approach would be to increase the bending field to $5 \mathrm{~T}$ at three locations in the storage ring with superconducting dipoles (Superbends) to generate photons with a critical energy around $12 \mathrm{keV}$.

A Superbend R\&D program was carried out which culminated in a successful magnet that did not quench before reaching critical current. It could be ramped to full field in less than $100 \mathrm{sec}$ while submerged in liquid helium and had acceptable field quality [2]. This magnet was used as the starting point for the design and fabrication of the accelerator-grade Superbend magnet system described in this paper. The detailed design and fabrication is underway at Wang NMR, under contract to LBNL. We report preliminary test results for the first of four Superbend magnets.

\footnotetext{
Manuscript received September 18, 2000. This work was performed at the Lawrence Berkeley National Laboratory with the support of the Director, Office of Science, Office of High Energy and Nuclear Physics, High Energy Physics Division, U. S. Department of Energy under Contract No, DE-AC0376SF00098.

${ }^{\dagger}$ Lawrence Berkeley National Laboratory, 1 Cyclotron Road, Berkeley, CA 94720 USA. Author addresses are available at www.lbl.gov.

${ }^{\dagger \dagger}$ Wang NMR, 550 North Canyons Parkway, Livermore, CA 94550 USA.
}

\section{A. Magnetic Requirements}

The detailed magnetic requirements are contained in [3] and [4]. The selected requirements listed in Table I are derived from ALS accelerator physics considerations. All Superbend magnets are required to be nearly identical, so rather tight construction tolerances are placed on the magnetic components.

TABLE I

SUPERBEND MAGNETIC REQUIREMENTS

\begin{tabular}{ll}
\hline \hline \multicolumn{1}{c}{ Quantity } & \multicolumn{1}{c}{ Value } \\
\hline Central Field & $5.69 \mathrm{~T}$ \\
Field Integral $(1.9 \mathrm{GeV})$ & $1.105 \pm 0.1 \% \mathrm{~T}-\mathrm{m}$ \\
Sextupole (systematic) & $\leq 60 \times 10^{-4}$ at $1 \mathrm{~cm}$ \\
Higher Order & $\leq 2 \times 10^{-4}$ at $1 \mathrm{~cm}$ \\
Sextupole (random) & $\leq 3 \times 10^{-4}$ (peak to peak) at $1 \mathrm{~cm}$ \\
Longitudinal Position & Within $\pm 1 \mathrm{~mm}$ of ideal \\
Transverse Position & Within $\pm 0.5 \mathrm{~mm}$ of ideal \\
Vertical Position & Within $\pm 0.4 \mathrm{~mm}$ of ideal \\
Roll & $\leq 250 \mu \mathrm{rad}$ \\
Longitudinal Vibration & $\leq 3 \mu \mathrm{m}>0.01 \mathrm{~Hz}$ \\
Transverse Vibration & $\leq 5 \mu \mathrm{m}>0.01 \mathrm{~Hz}$ \\
Vertical Vibration & $\leq 5 \mu \mathrm{m}>0.01 \mathrm{~Hz}$ \\
Rolling Motion & $\leq 2 \mu \mathrm{rad}>0.01 \mathrm{~Hz}$ \\
\hline
\end{tabular}

Table II lists the operational goals, which will minimize the impact of the Superbends on ALS operation. These were derived from accelerator physics considerations [4].

TABLE II

SUPERBEND OPERATIONAL GOALS (IN ORDER OF PRIORITY)

\begin{tabular}{ll}
\hline \hline \multicolumn{1}{c}{ Quantity } & \multicolumn{1}{c}{ Value } \\
\hline Field Ripple & $\leq 5 \times 10^{-6}$ for frequencies $>0.01 \mathrm{~Hz}$ \\
& $\leq 1.7 \times 10^{-5}$ for frequencies $\leq 0.01 \mathrm{~Hz}$ \\
Field Tracking & $\approx 300 \mathrm{~mA}$ at $1 \mathrm{~A} / \mathrm{s}$ \\
Ramping Rate & $1.5 \mathrm{~A} / \mathrm{s}$ to match present ALS operation \\
(204A to 291 A) & $3 \mathrm{~A} / \mathrm{s}$ to match ALS possible upgrade \\
\hline
\end{tabular}

\section{B. Coil and Cold Mass Design}

The conductor specified for the ALS Superbend was developed in the R\&D program [2] and was supplied by Outokumpu per the parameters in Table III. 
TABLE III

SUPERBEND CONDUCTOR PARAMETERS

\begin{tabular}{ll}
\hline \multicolumn{1}{c}{ Quantity } & \multicolumn{1}{c}{ Value } \\
\hline Type & Monolithic, NbTi/Cu \\
Cu:NbTi ratio & 3.0 \\
Number of filaments & 330 \\
Filament Diameter & 39 microns \\
Twist Pitch & $26 \pm 2 \mathrm{~mm}$ \\
Insulation & Formvar \\
Dimensions (bare) & $0.9 \mathrm{~mm} \times 1.8 \mathrm{~mm}$ \\
Dimensions (insulated) & $1.00 \mathrm{~mm} \times 1.90 \mathrm{~mm}, \pm 0.02 \mathrm{~mm}$ \\
$\mathrm{I}_{\mathrm{c}} @ 4.25 \mathrm{~K}, 5 \mathrm{~T}(0.1 \mu \mathrm{V} / \mathrm{cm})$ & $1110 \mathrm{~A}$ \\
RRR & 200 \\
\hline
\end{tabular}

The Superbend is conduction cooled, in order to place the coils as close to the ALS electron beam as possible. To make the Superbend operation in the ALS as automatic, efficient, and economical as possible we use a 2-stage GiffordMcMahon cryocooler to provide the required refrigeration [5]. The $4 \mathrm{~K}$ heat load is minimized by the use of HTS leads, supplied by American Superconductor Corp., from $50 \mathrm{~K}$ to 4 $\mathrm{K}$, and epoxy-fiberglass suspension straps with $50 \mathrm{~K}$ heat stations. Conduction-cooled copper current leads from room temperature to $50 \mathrm{~K}$ are used. The design heat loads are tabulated in Table IV. We selected the Sumitomo Model SRDK-415 cryocooler after a series of cryogenic tests [6].

To continue operation in case of cryocooler failure, we include an 85-liter liquid helium vessel and a 35-liter liquid nitrogen vessel inside the Superbend vacuum vessel. We will have about 18 hours of running before we must supply cryogens from external dewars.

TABLE IV

SUPERBEND DESIGN HEAT LOADS

\begin{tabular}{lcc}
\hline \multicolumn{1}{c}{ Source } & $\begin{array}{c}\text { Heat Load to } 50 \mathrm{~K} \\
(\mathrm{~W})\end{array}$ & $\begin{array}{c}\text { Heat Load to 4 K } \\
\text { (W) }\end{array}$ \\
\hline Current Leads & 27 & 0.25 \\
Thermal Radiation & 6.7 & 0.03 \\
Magnet Supports & 1.1 & 0.06 \\
Tubes to LN Reservoir & 1.9 & 0 \\
Tubes to LHe Reservoir & 0.9 & 0.06 \\
Rapid Cool Down Tubes & 0.8 & 0.04 \\
Instrumentation wires & 0.1 & 0.001 \\
\multicolumn{1}{c}{ Total } & 38.5 & 0.44 \\
Cryocooler Capacity & 45 & 1.5 \\
\hline
\end{tabular}

Fig. 1 shows the essential features of the Superbend coil and cold mass assembly. The superconducting coils have laminated AISI 1006 steel poles and an outer ring of aluminum alloy 5083-H321 for structural support. In order to provide conduction cooling to the coils, each coil is surrounded by a high-purity copper coil form that is thermally connected to the LHe vessel and cryocooler second stage. The coils are precisely mounted to the C-shaped return yoke, laminated AISI 1006 steel, with bolts and locating pins.

In order to provide a large enough room temperature gap (see Fig. 2), the distance between the iron poles was increased to $100 \mathrm{~mm}$, about $6 \mathrm{~mm}$ larger than in the R\&D magnet [2]. Table $\mathrm{V}$ contains relevant details of the Superbend magnet.
TABLE V

SUPERBEND MAGNET PARAMETERS

\begin{tabular}{ll}
\hline \hline \multicolumn{1}{c}{ Quantity } & \multicolumn{1}{c}{ Value } \\
\hline Magnet type & Racetrack windings, iron poles \\
Pole length along beam & $114 \mathrm{~mm}$ \\
Pole length transverse to beam & $180 \mathrm{~mm}$ \\
Turns per layer & 33 \\
Number of layers & 70 \\
Conductor length per coil & $1725 \mathrm{~m}$ \\
Operating current & $291 \mathrm{~A}$ at $1.9 \mathrm{GeV}$ \\
Peak field at conductor & $6.8 \mathrm{~T}$ \\
Fraction of critical current & 0.44 at $4.3 \mathrm{~K}$ \\
Stored energy & $150 \mathrm{~kJ}$ \\
Low-field inductance & $11 \mathrm{H}$ \\
High-field inductance & $3 \mathrm{H}$ \\
Total cold mass & $1500 \mathrm{~kg}$ \\
\hline
\end{tabular}

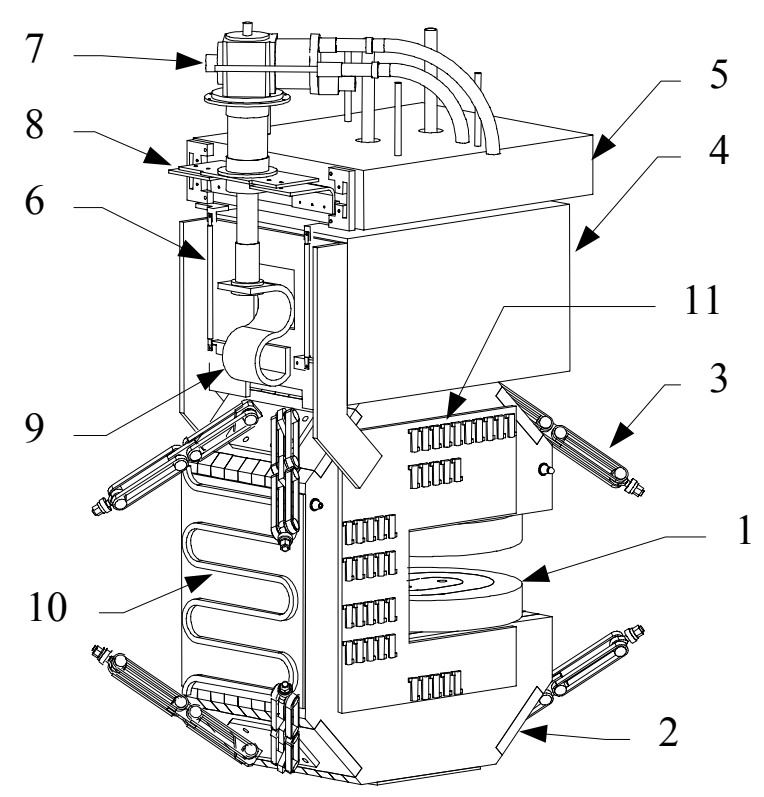

Fig. 1. Superbend cold mass assembly: 1 - superconducting coils with steel poles, 2 - laminated steel yoke, 3 - suspension straps, 4 - LHe vessel, 5 - $\mathrm{LN}_{2}$ vessel, 6 - HTS leads, 7 - cryocooler, 8 - $50 \mathrm{~K}$ thermal connection, 9 $-4 \mathrm{~K}$ thermal connection, 10- cooldown tube, 11 -warmup heater.

The LHe vessel, made from 304L stainless steel, is bonded to the steel yoke with low-melting point solder to provide reasonable thermal contact. The vessel contains an internal copper heat exchanger, connected to the cryocooler second stage, to allow the helium to be continually recondensed.

The $4 \mathrm{~K}$ cold mass (coils, yoke, and LHe vessel) is suspended with 8 straps as shown in Fig.1. The straps are made from epoxy and unidirectional S-glass fibers for low heat load, and are designed for 2-g transportation loads in addition to the static magnet support loads. The cold mass position can be adjusted with nuts on the outside of the vacuum vessel as indicated on Fig. 2. Instrumented load washers measure the strap tension to ensure that the strap loads are roughly equal during adjustment. With this suspension, the center of mass does not move upon cooldown.

Tubes attached to the steel yoke allow rapid cooling with liquid nitrogen to $100 \mathrm{~K}$ and liquid helium to operating 
temperature. The goal is to cool from room temperature to operating in about 8 hours.

A warmup heater will supply $2.8 \mathrm{~kW}$ to the steel yoke when supplied with $200 \mathrm{~V}$. The goal is to warm from operating temperature to room in about 8 hours. The heater leads are internally disconnected with a linear motion feedthrough when not in use to minimize $4 \mathrm{~K}$ heat load.

Existing ALS storage ring components such as electron beam vacuum chamber, vacuum valves, photon stops and the tunnel shielding walls require the Superbend to have the configuration shown in Fig. 2.

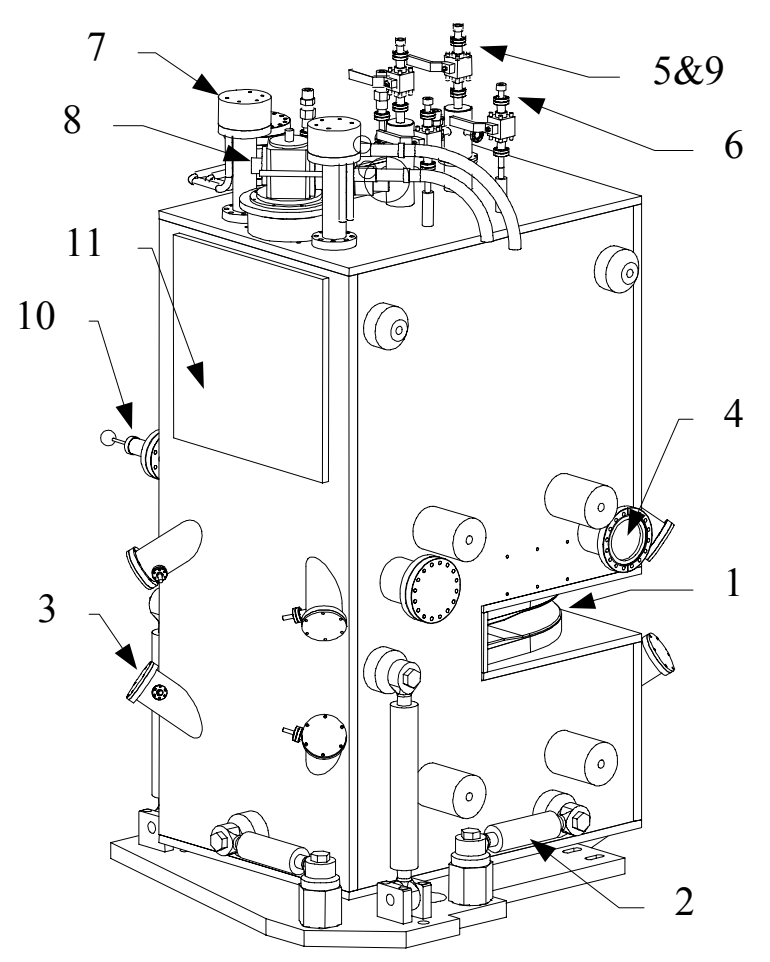

Fig. 2. Exterior configuration of the ALS Superbend: 1 - room temperature gap, 2 - suspension struts, 3 - cold mass suspension strap adjusters, 4 - cold mass fiducial viewport, 5 - LHe ports, $6-\mathrm{LN}_{2}$ ports, 7 current leads, 8 - cryocooler, 9 - rapid cooldown ports, 10 - rapid warmup feedthrough, 11 -access port.

The vacuum vessel is mounted to the ALS ring girder after being positioned over the ALS storage ring vacuum chamber. Clearance allows for magnet alignment with the 6-strut suspension system.

Items that were considered to require servicing and possible replacement, such as cryocooler, current leads, and quench protection diodes (see section C) are readily accessible by warming to room temperature and removing the access port

\section{Quench Protection, Instrumentation, and Power Supply}

The Superbend is equipped with a set of cold diodes to provide a passive means of rapidly discharging the coils in case of quench and protection in case of current lead failure. Fig. 3 shows the power and instrumentation schematic. Diagnostic wires measure coil voltages, HTS lead voltages, and diode protection system voltages.
In addition, the Superbend has eight Cernox temperature sensors from LakeShore Cryotronics. These measure the temperature of both cryocooler stages, the warm end of both HTS leads, the copper coil forms of both coils, and the center yoke plate above and below the beam aperture gap.

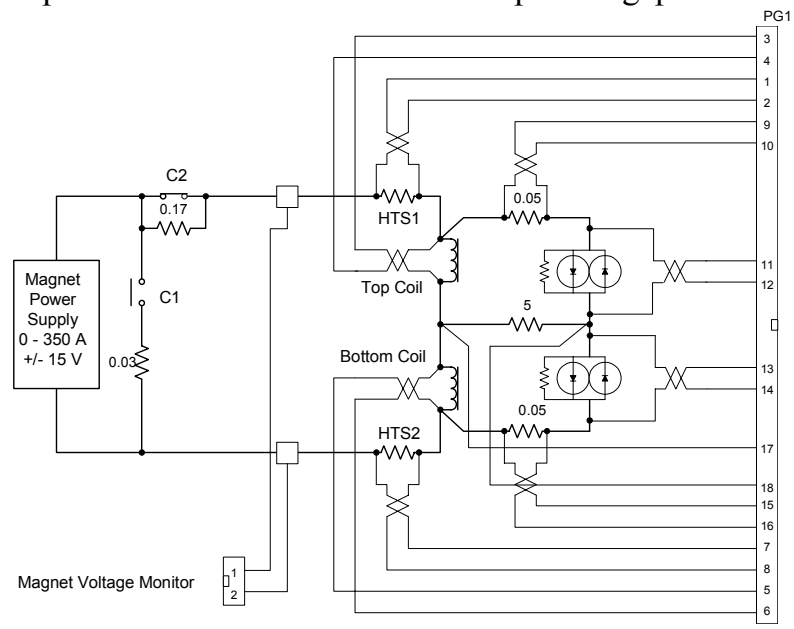

Fig. 3. Superbend power and instrumentation schematic.

Quench detection instrumentation (not shown) monitors the voltage across each of the coils and HTS leads, and the total voltage across the two magnet coils using high quality op-amps. These signals are lowpass filtered and applied to a bank of threshold detectors that will send a fault signal to the power supply if their threshold is exceeded. The fault status is latched and displayed on the front panel and made available to the accelerator control system.

The power supply was constructed by the Dynapower Corporation to meet the requirements of Table II [7]. The power supply input is three phase $208 \mathrm{~V}$ AC, rectified by Silicon Controlled Rectifiers (SCR) on the secondary side of the transformer. The 6-pole rectified DC voltage is filtered with a passive component network. The output current is sensed with a high quality Zero Flux Current Transformer (ZFTC), which is used as a feedback element to control the phase angle of the SCRs and consequently the output current. The power supply has an output of $350 \mathrm{~A}, \pm 15 \mathrm{~V}$ (quadrants 1 and 4) to provide for charging and discharging the Superbend.

The current-dependent inductance of the Superbend (Table V) is compensated in the electronic circuitry of the feedback loop. The resistor and capacitor components in the compensation network are chosen to minimize the overshoot in the output current after the current ramp rate is changed, while maintaining the stability performance of the power supply.

The power supply is equipped with two types of external fault interlocks. The first results in a discharge with a time constant of $\sim 100 \mathrm{~s}$, which will not quench the Superbend. The second results in a faster discharge with a time constant of $\sim 20 \mathrm{~s}$ to protect the HTS leads. This may cause the Superbend to quench. 


\section{Fiducialization and Alignment}

The pole tips, return yoke and vacuum vessel have features to enable the ALS Survey team to position the cold mass close to the correct position in the cryostat using optical tooling.

Final positioning is done with the 6-strut suspension, based on magnetic measurements of the quadrupole fields that define the magnetic center. Magnet roll is determined by the ratio of skew to normal dipole components. Our roll measurement resolution is less than the required tolerance, so a trim coil set is incorporated in the design to provide beambased roll adjustment to the required value.

\section{TEST REsults}

Tests are underway on SBM1, the first Superbend, and we report our preliminary results in this section.

\section{A. Magnet Powering}

Initially, an electronic glitch traced to a grounding problem caused a trip at 299.2 A, but soon after SBM1 was charged to $302 \mathrm{~A}$ at $0.1 \mathrm{~A} / \mathrm{s}$ without quenching. SBM1 was then cycled between $200 \mathrm{~A}$ and $300 \mathrm{~A}$ at rates up to $\pm 1 \mathrm{~A} / \mathrm{s}$ without quenching. Acceptance testing continues to determine the limiting ramp rate and verify compliance with the specification.

After empirical adjustment of the compensation network, we verified that the power supply meets specification and is suitable for ALS operation. Fig. 4 shows the satisfactory response of the power supply output current to a ramped control voltage.

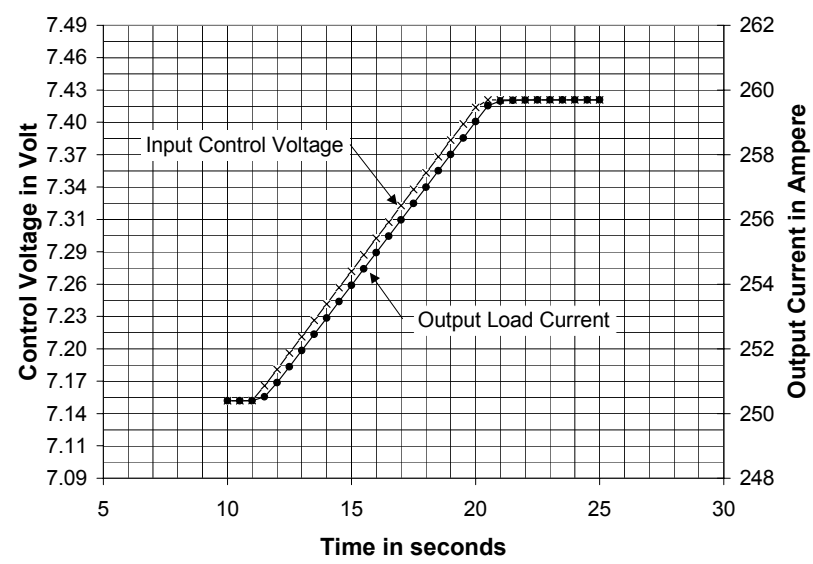

Fig. 4. Superbend power supply output current vs. control signal corresponding to a $1 \mathrm{~A} / \mathrm{s}$ ramp rate.

\section{B. Magnetic Measurements}

The rotating coil used in [2] is also used to perform magnetic measurements on the Superbend. A calibrated Hall probe is used to measure the peak central field. Table VI summarizes the present status of the magnetic measurements. The results appear to be within specification.

\section{Vibration Testing}

Cold mass motion induced by the cryocooler was measured with Model 731A seismic accelerometers from Wilcoxon Research. The measurements revealed that the $4 \mathrm{~K}$ thermal connection was the main pathway for induced vibrations and a design change was implemented which reduced the vibrations well below the values in Table I. The $50 \mathrm{~K}$ thermal connection and the vacuum bellows played no significant role in cold mass motion.

In addition, other measurements were carried out in the ALS tunnel which verified that transmission of cryocooler vibrations to neighboring magnets is not a problem [8].

TABLE VI

SBM1 MAGNETIC MEASUREMENTS AT 298.9 A

\begin{tabular}{lcc}
\hline \hline \multicolumn{1}{c}{ Quantity } & $\begin{array}{c}\text { Measured } \\
\text { Value }\end{array}$ & Specification Value \\
\hline Central field, B & $5.738 \mathrm{~T}$ & $5.69 \mathrm{~T}$ \\
Integrated dipole & $1.119 \mathrm{~T}-\mathrm{m}$ & $1.107 \mathrm{~T}-\mathrm{m}$ \\
Normal Quadrupole & $1.022 \times 10^{-3}$ (a) & $<5 \times 10^{-4}$ (b) \\
Skew Quadrupole & $1.320 \times 10^{-4}$ & $<6 \times 10^{-4}$ \\
Normal Sextupole & $1.732 \times 10^{-3}$ & $<6 \times 10^{-3}$ \\
Higher multipoles & $<3 \times 10^{-5}$ & $<2 \times 10^{-4}$ \\
Horizontal Offset & $3.8 \mathrm{~mm}$ & $<1.9 \mathrm{~mm}^{(\mathrm{c})}$ \\
Vertical Offset & $0.4 \mathrm{~mm}$ & $<2.3 \mathrm{~mm}^{(\mathrm{c})}$ \\
\hline
\end{tabular}

a. May require horizontal adjustment of Superbend.

b. Magnet to magnet variation.

c. Based on measured multipoles. Horizontal adjustment range is $\pm 10 \mathrm{~mm}$.

\section{Thermal Performance}

Based on earlier measurements of heat input and cryocooler temperature [6], we believe the heat loads in SBM1 exceed the design values given in Table IV. We estimate the stage $1(50 \mathrm{~K})$ heat load to be about $50 \mathrm{~W}$ and the stage $2(4 \mathrm{~K})$ heat load to be about $1.1 \mathrm{~W}$. We suspect the increased heat load is in the beam aperture region, where space is very tight. SBM1 may be useable in the ALS as it stands, but we plan to inspect the magnet after we have completed more tests and correct the problem.

\section{ACKNOWLEDGMENT}

The authors wish to thank Kurtis Nishimura, Dan Colomb, and Dennis Calais for their assistance in carrying out the vibration testing.

\section{REFERENCES}

[1] C. E. Taylor and S. Caspi, "A 6.3 T Bend Magnet for the Advanced Light Source”, IEEE Trans. Magnetics 32, No. 4, 1996, pp. 2175-2178.

[2] C. E. Taylor, et al., "Test of a High-Field Magnet for the ALS," Transactions Applied Superconductivity 9, No.2, 1999, pp. 479-482.

[3] Egon Hoyer and Jon Zbasnik, "Superbend Magnet Specification", Lawrence Berkeley National Laboratory Specification M909, Nov. 1999.

[4] Advanced Light Source Division, "Superbend Magnet System Conceptual Design Report", Lawrence Berkeley National Laboratory Publication, PUB-5457, Apr. 2000.

[5] M. A. Green, et al., "Refrigeration options for the ALS Superbend dipole magnets", to be published in proceedings of the 1999 CEC, Montreal, Quebec, Canada, July 1999.

[6] J. Zbasnik, M. A. Green, E. H. Hoyer, C. E. Taylor, J. Y. Chen, and S. T. Wang, "Tests of a GM cryocooler and high Tc leads for use on the ALS Superbend magnets", ibid.

[7] G. J. DeVries, "Superbend magnet power supply specification," Lawrence Berkeley National Laboratory Specification LSE-128.

[8] C. Steier, private communication. 